\title{
Investigation of electrostatic fields by modeling methods
}

\author{
Alla Merkulova ${ }^{1, *}$, Irina Cherunova $^{1}$, Irina Danilenko $^{1}$ \\ ${ }^{1}$ Don State Technical University, Rostov, Russia
}

\begin{abstract}
Annotation. The environment is filled with electrostatic fields of various origins. The negative influence of the electrostatic field requires the provision of electrostatic safety standards. So modeling of electrostatic fields is a topical research task. The electrization of objects is complex, depending on the many parameters process. The article considers the existing methods of studying electrostatic fields. The authors have justified the criterion for assessing the safety of an electrostatic field. The article proposes the developed advanced mathematical model describing the electrization of a complex, multi-layered object. Variable values in the proposed mathematical model are the main characteristics of the materials forming the layers of the modeled object, and the parameters of the condition of the environment. The constructed mathematical model of electrization of a complex object has undergone a successful experimental verification in field trials. Analysis of the experimental data confirmed the expected condition of the system, which is a complex object.
\end{abstract}

\section{Introduction}

The investigation of electrostatic fields surrounding human in everyday life and at work is a topical scientific task. Active use of the electric current energy leads to the formation of electrical charges on the external elements of household appliances and production structures. Another reason for static electricity charges on objects used by a person is the use of new materials. Modern materials are materials that are complex in composition and structure, which, as a general rule, have high electrical resistance. Surface electric charge appears on household items. Surface electric charge appears even on clothing. Electrization of materials in this case occurs during the operation of products.

Electric charge is a negative production factor. The limiting values of the parameters determining the magnitude of the electric charge are limited by normative documents [1].

Spark gas discharge is one of the processes of the accumulated electric charge from the surface of the material. Spark gas discharge can cause primary injuries associated with the passage of electric current through the human body. They include: a burn with a spark of human skin, the ignition of hazardous substances in the environment. A huge temperature (up to $20000 \mathrm{~K}$ ) is concentrated in the spark discharge channel. This powerful heat flow causes the instantaneous ignition of the materials of the clothing package, severe burns and is the main damaging factor when exposed to an electric discharge directly on a person. Spark gas discharge can cause secondary injuries. They include: bruises and injuries in the fall. In this case, even weak discharge currents can cause a breakdown in the nervous system and worsen a person's overall condition, if their action doesn't stop for a long time.

As is known, the control system of a living organism is built on neuro-electrical impulses. Therefore, the effect of an external electrostatic field on a living organism is very complex and can cause functional disorders of the nervous and cardiovascular systems.

However, the main danger of static electrization is associated with discharges of static electricity. Static electricity discharges can ignite flammable gases, vapors or dust in the air. Therefore, in many enterprises, the conditions of a person's professional activity are associated with an electrostatic danger [2]. For example, at enterprises of the oil and gas complex, the environment contains flammable liquids, their vapors and gases. The requirements to ensure for a person safe living and working conditions [3, 4] determine the direction of scientific researches. Therefore, the investigation of the conditions for the appearance and change of the characteristics of electrostatic fields is a topical scientific task.

\section{Similarity method for the study of electrostatic fields}

The similarity method is often used in the study of electrostatic fields. This method is a physical simulation of the phenomenon. When studying real electrostatic fields, equivalent fields are created. For example, the electrostatic field between charged conductive bodies replaces the study of the current field between the same

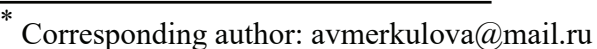


system of conductors. The electrical potentials of the conductors are maintained constant.

The similarity method asserts that if the sizes of the electrodes creating the electrostatic field and all the distances between these electrodes vary in the same proportion, the structure of the electrostatic field will remain the same.

Electrostatic fields in electrostatic lenses, in electronic tubes are very complex and unique electrostatic fields. Such electrostatic fields do not describe analytically. When studying similar electrostatic fields, the similarity method is used. The similarity method is rationally used in describing the electrostatic fields of stable electrified systems.

Such a study is experimental. In this case, by means of electrical measuring instruments, a large number of differences in the space in which the electrostatic field is distributed are measured. Then the researchers build the surfaces of an equal potential and the line of tension of the electrostatic field under study. Visualization of the results of the research is usually carried out with the help of special programs for computer simulation of electric fields.

However, the similarity method allows studying only the given electrostatic field created by a real system of charged bodies and at a particular time. In this case, it is impossible to make estimates of the dynamics of the change in the parameters of the electrostatic field. The similarity method does not allow to use the received scientific information on similar electrified systems, the parameters of which differ slightly.

\section{Modeling of electrification processes}

In everyday life and production human uses materials, most of which are dielectrics. They are electrified by contact with each other and with conductors.

On the surface of the dielectric, an electric charge is accumulated. It is practically impossible to reduce the electric charge accumulated on the dielectric surface. As a result of these processes, an electrostatic field is created in the surrounding electrified materials.

The processes of the appearance of electric charge and the influence of electrostatic fields on production and on the human body were considered in the works of a number of researchers [5-7].

Researcher Loeb [7] notes the difficulty in interpreting and comparing the experimental results obtained in the study of electrization. He explains the emerging problem by the variety of processes of interaction between contacting bodies and material environments into which materials are placed. Friction, compression, stretching, shearing, etc., increase the contact area of two bodies. Therefore, the rate of exchange processes changes and the value of the electrostatic charge formed on the contacting surfaces increases. All researchers note the great role of mechanical influences in electrization processes.

Investigations of the contact electrization of materials have shown that the contact of clean surfaces in the process of electrization is played by electrons. If materials are contaminated or active friction occurs, in the process of electrization the main role is played by ions or charged particles of matter. The resultant surface charge density is determined by the type of charge carriers.

The model of the electrization process at the contact of two dielectrics with different energies of the Fermi level is provided by the electron transition and considered in [8]:

$$
\sigma= \pm \frac{1,77 \cdot 10^{-9}(\Delta \Phi-\Delta \Psi)}{\mathrm{e}\left(\mathrm{v}_{1} / \varepsilon_{2}+\mathrm{v}_{2} / \varepsilon_{1}\right)},
$$

where $\sigma-$ is the surface electric charge, $\mathrm{C} / \mathrm{m}^{2} ; \Delta \Phi-$ is the difference of energy at the Fermi level, eV; $\Delta \Psi$ - is the difference in the electron affinity energy, eV; e - is the value of the elementary electric charge, $C ; v_{1}$ and $v_{2}$ - the depth of injection of charge carriers with respect to the interface of dielectrics (for the first and second material, respectively), $\mathrm{m} ; \varepsilon_{2}$ and $\varepsilon_{1}-$ are the dielectric permittivities of the contacting materials (respectively, the first and second substances).

The model of the electrification process, based on the transition of ions from one surface to another, was proposed in [9]:

$$
\sigma=\frac{\varepsilon_{0} \varepsilon}{\mathrm{h}}\left[\left(\mathrm{W}_{1}-\mathrm{W}_{2}\right)-\frac{\mathrm{kT}}{\mathrm{e}} \ln \frac{\mathrm{n}_{1}}{\mathrm{n}_{2}} \pm \mathrm{hE}\right]
$$

where $\varepsilon_{0}$ - is the electric constant (equal to $8,85 \cdot 10^{-12}$ $\mathrm{F} / \mathrm{m}) ; \varepsilon$ - is the minimum of two $\left(\varepsilon_{2}\right.$ and $\left.\varepsilon_{1}\right)$ dielectric permittivity; $\mathrm{h}$ - distance between surfaces (unevenness height), $\mathrm{m} ; \mathrm{W}_{1}$ (and $\mathrm{W}_{2}$ ) - is the binding energy of charge carriers to a solid, eV; $\mathrm{k}$ - is the Boltzmann constant (equal to $1,38 \cdot 10^{-23} \mathrm{~J} / \mathrm{K}$ ); $\mathrm{T}-$ is the absolute temperature, $\mathrm{K} ; \mathrm{n}_{1}\left(\right.$ and $\left.\mathrm{n}_{2}\right)$ - is the concentration of ions or particles, $\mathrm{m}^{-3} ; \mathrm{E}-$ external electrostatic field strength, $\mathrm{V} / \mathrm{m}$.

Static electrization consists of two processes: the accumulation of static electric charges and the draining of electric charges over time. If the processes of generation of electric charges and their decay are continuous, then one can go to infinitesimals and integrate:

$$
\sigma(t)=\int \frac{d \sigma_{\text {рост }}}{d t} d t-\int \frac{d \sigma_{\text {спад }}}{d t} d t
$$

Experiments show that the process of electric charge flow can be considered continuous. The process of the flow of electric charge is determined by the electrical conductivity of the material of the electrified object and the emission of a charge from its surface into the environment. Generation of electric charges is usually a step-by-step process. Generation of electric charges is determined by friction, compression of contacting surfaces during electrization. When modeling the standard processes of object electrization, it is possible to generate and drain the electric charge continuously. 


\section{Construction of a model for the electrization of a multilayered package of materials}

From the electrization processes, electrization by friction is more common. Electrization by friction is accompanied by friction, squeezing and deformation. Analysis of the developed models of electrization showed that they are studying, as a rule, contact electrification. We have considered a more complicated process of electrization. Our model studies the electrization of a multilayered object taking into account friction, deformation and emission of electric charge into the environment [10].

The friction process leads to an increase in temperature at the points of contact. The increase in temperature leads to an increase in the rate of metabolic processes, including electrization. When the contacting objects are squeezed, deformation of their surfaces arises. The deformation of the surfaces leads to an increase in the contact area of the surfaces. The growth of the area of contact increases the degree of electrification of objects. Emission of electric charge is determined by the electrical conductivity of the environment. The emission of electric charge reduces the value of the electric charge accumulated on the surfaces of the contacting objects.

The development of common numerical algorithms that make it possible to calculate three-dimensional electrostatic fields with the necessary accuracy is an topical research task. The problem of constructing models of electrostatic fields arises for inhomogeneous and nonlinear material environment and complex forms of interfaces for material environment.

We considered triboelectrization of a multilayer object. In the proposed model of electrization, we assessed the contribution to the electrification process of a complex object of electrostatic charges accumulated by separate surfaces of structural layers.

The electrostatic field is a potential force field. At each point in space, the electrostatic field is determined by the values of the tension vector $\vec{E}$ and the potential $\varphi$ of the electrostatic field.

Restrictions on occupational safety [11] require that, $\mathrm{W} \leq \mathrm{kW}_{\min }$, where $\mathrm{W}_{\min }-$ minimum energy of ignition of gas mixtures, $\mathrm{k}$ - safety factor $(\mathrm{k}=0,4)$.

The limiting values of the parameters of the electrostatic field in the air are subject to Paschen's law [12]. The energy of the electrostatic field is given by:

$$
\mathrm{W}=\frac{\varepsilon \varepsilon_{0} \mathrm{E}^{2} \mathrm{Sd}}{2},
$$

where $\mathrm{E}$ - is the intensity of the electrostatic field, $\mathrm{V} / \mathrm{m}$.

It's obvious that $\mathrm{E} \sim \sqrt{\mathrm{W}}$. This is the rationale for choosing the parameter for assessing the electrostatic safety of environmental conditions. The value of the electric field strength can be used as a parameter for assessing the electrostatic safety of environmental conditions.
We have obtained an estimate of the critical value of the electric field strength: $\mathrm{E}_{\text {кр }} \sim \sqrt{0.4} \mathrm{E}_{\text {пр }}$, here $\mathrm{E}_{\mathrm{\pi p}}=3 \cdot 10^{6} \mathrm{~V} / \mathrm{m}$ is the limiting value of the electrostatic field strength for the air medium under normal conditions [5]. Thus, the critical value of the electric field strength is equal to $\mathrm{E}_{\mathrm{kp}}=1,90 \cdot 10^{6} \mathrm{~V} / \mathrm{m}$.

We obtained an analytical solution for calculating the characteristics of the electrostatic field formed by the electric charges distributed in space. We simplified the geometric form of the electrified bodies under study. In the mathematical model of the electrostatic field, we switched from real objects possessing non-linear characteristics to an ideal one, i.e. linear. The external environment in which the electrostatic charges that create the electrostatic field are distributed is inhomogeneous. We used the method of secondary sources to move to a homogeneous medium. Tozoni [13]. has developed the basis of the secondary sources method. The method of secondary sources introduces additional bound charges, the values of which are determined by the characteristics of the contacting surfaces and the parameters of the media separating them. We moved to a homogeneous medium, adding additional secondary sources distributed along the media interface.

In our mathematical model of electrification, the surface electric charge of the i-th layer of a multilayer object is determined by the equation:

$$
\sigma_{\mathrm{i}}(\mathrm{t})=\sigma_{\text {фрик_i }} \exp \left(-\mathrm{t} / \varepsilon_{0} \varepsilon \rho_{\mathrm{V}}\right)-\sigma_{\text {эм }}
$$

here $\sigma_{\text {фрик_i }}-$ is the value of the generated surface electrostatic charge (determined by the refined (1)

$$
\sigma_{\text {фрик }}=\frac{\mathrm{a} \varepsilon_{0} \varepsilon}{\mathrm{h}}\left[\left(\mathrm{W}_{1}-\mathrm{W}_{2}\right)-\frac{\mathrm{kbT}}{\mathrm{e}} \ln \frac{\mathrm{n}_{1}}{\mathrm{n}_{2}} \pm \mathrm{hE}\right]
$$

here the coefficients $\mathrm{a}$ and $\mathrm{b}$ take into account friction and compression, respectively; $\sigma_{э м}-$ surface electric charge flowing as a result of emission into the environment.

Now the value of the resultant surface electric charge on the surface of the i-th layer of the multilayer object is determined by the equation:

$$
\sigma_{\mathrm{i}}=\sigma_{\mathrm{cB}_{-} \mathrm{i}}+\sigma_{\mathrm{i}}(\mathrm{t})
$$

here $\sigma_{\text {св }{ }_{i}}-$ the surface electric charge created by injected padding latent electric charges.

Electrostatic intensity, created by a multilayer electrified object, in any point of space is defined by the equation:

$$
\mathrm{E}_{\mathrm{n}}=\frac{1}{\varepsilon_{0} \varepsilon \mathrm{R}} \sum_{\mathrm{i}=1}^{\mathrm{N}} \sigma_{\mathrm{i}} \mathrm{r}_{\mathrm{i}}
$$

The intensity of the resultant electrostatic field is:

$$
\overrightarrow{\mathrm{E}}=\overrightarrow{\mathrm{E}}_{\mathrm{n}}+\overrightarrow{\mathrm{E}}_{\text {внеш }}
$$


here $\overrightarrow{\mathrm{E}}_{\text {внеш }}$ - is the strength of the external electric field.

The safety requirements limit the modulus of the resulting electric field E. The strength of the electric field should not exceed the critical value $\mathrm{E}_{\mathrm{kp}}$.

The proposed improved mathematical model of the electrization process is described by a system of equations (2-6). The main characteristics of the materials of the layers of the simulated complex object and the parameters of the state of the environment are variable values in this system of equations $[14,15]$.

This system of equations simulates the electrization of a multilayer object. Each layer of the object is materials with different characteristics. Researchers and engineers can evaluate the modulus of the electrostatic field strength near the outer surface of a complex object. The proposed mathematical model of the electrization process allows us to predict the provision of electrostatic safety in the vicinity of the object under study.

\section{Conclusion}

The constructed mathematical model of the electrization of a complex object has undergone a successful experimental verification in tests [16]. We conducted experiments on the electrization of a multilayered package of heat-protective clothing [15]. We estimated the magnitude of the electrostatic field strength modulus near the surface of the heat-shielding suit. We subjected the triboelectrization in real conditions of operation to a heat-protective suit.

Analysis of the experimental data confirmed the expected condition of the heat-shielding suit. Preliminary mathematical modeling of the process of electrifying a heat-shield suit should be carried out when constructing a costume design. Preliminary mathematical modeling of the process of electrization a heat-protective suit will help to select materials for it. Preliminary mathematical modeling of the process of clothing electrization will provide a stable protection of a person from static electricity.

The results of optimization of the design of a special anti-electrostatic suit for protection from low temperatures based on the mathematical model of electrization of a complex object, developed by us, are confirmed by patents [17-20].

\section{References}

1. GOST 12.0.003-2015 Occupational safety standards system. Dangerous and harmful working factors, Date of issue 2017-03-01, (Standartinform, Moscow, 2016)

2. V.A Veryovkin, V.I. Gorshkov, V.A. Bondar, B.G. Popov, Static electricity in polymers: reports of a seminar "A static electricity in polymers», 42-45, (Chemistry, Leningrad, 1968).

3. GOST 12.4.045-84 Occupational safety standards system. Electrostatic fields. Tolerance levels and methods of control at working places Date of issue 30.06.85. - (Standartinform, Moscow, 1985)
4. GOST 12.4.124-83. Occupational safety standards system. Means of the protection against static electricity . General technical requirements Date of issue 01.01.1984. (Standards Publishing House, Moscow, 1983)

5. P.L. Gefter, The electrostatic phenomena in processes of processing of chemical fibers (Legprombytizdat, Moscow, 1989).

6. G.A. Lushcheykin, Research techniques of electric properties of polymers (Chemistry, Moscow, 1988)

7. L. Loeb Static electrization Gosenergoizdat (Moscow-Leningrad, 1963)

8. D.R. Davis, Inst. Phys. Conf. Ser., N 4, 29-36. (1963)

9. P. S. Henry, H. J. Text. Inst., N 1., 48, $1-7$ (1957)

10. Ch.M. Badmayev, etc. The Modern problems of science and education, №6., available at: http://www.science-education.ru/106-7972 (2012)

11. GOST 12.1.044-89 Occupational safety standards system. Fire and explosion hazard of substances and materials. Nomenclature of indices and methods of their determination Date of issue 01.01.1991. (Standards Publishing House, Moscow, 1989).

12. L. D. Landau, E.M. Lifshits, Theoretical physics in 10 volumes. Volume 8. Electrodynamics of continuous mediums: manual, Fizmatlit, Moscow, 2005)

13. O.V. Tozoni, Mathematical methods for calculation of electric and magnetic fields: (Naukova dumka, Kiev, 1964).

14. O.V., Kononova, A.V. Merkulova, I.V. Cherunova The Modern high technologies, №8., 1, 31-32 (2013)

15. A.V. Merkulova, Research and development of express anti-electrostatic clothes for protection against the under temperatures: abstract of Candidate of Technical Sciences, Shakhty (2007)

16. A.V. Merkulova, I.V. Cherunova, I.N. Danilenko, Measurement Techniques, 52, №4, 402-404 (2009)

17. I.V. Cherunova, A.V. Merkulova, I.Ju. Brink, V.V. Gorchakov, Patent № 2355266 Russian Federation, C2, MPK A 41D 13/008 Date of publication 20.05.2009 Bull. №14. (2006.01).

18. E.A. Shchenikova, I.V. Cherunova, A.V. Merkulova Patent № 2426483 Russian Federation, C1, MPK A 41D 13/008 Date of publication 20.08.2011 Bull. №23. (2006.01).

19. E.A. Shchenikova, I.V. Cherunova, A.V. Merkulova N.V. Kornev, The useful model № 128969 Russian Federation, U1, MPK A41D 13/008 Date of publication 20.06.2013 Bull. № 17. (2006.01).

20. E.A. Shchenikova, I.V. Cherunova, A.V. Merkulova N.V. Kornev, The useful model № 128968 Russian Federation, U1, MPK A41D 13/008 Date of publication 20.06.2013 Bull. № 17. (2006.01). 